\title{
Physical activity among 6o-69-year-olds in England: knowledge, perception, behaviour and risk factors
}

\author{
MOUSHUMI CHAUDHURY* and NICOLA SHELTON*
}

\begin{abstract}
This paper examines the risk factors that influence physical activity levels among a representative sample of older people in England and their awareness of the Chief Medical Officer's recommended level of physical activity. The paper analyses data from the cross-sectional, nationally-representative Health Surveys for England (HSfE) in 2006 and 2007. In HSfE20o6, I,550 adults aged 6o-69 years responded to a physical activity participation questionnaire, and in HSfE2007, 56I adults aged 60-64 years were asked about their knowledge of the physical activity requirement and their attitudes to participation. Very few respondents knew the recommended physical activity target, but more than one-half thought they had enough physical activity in their daily life, and over three-quarters thought they were 'very' or 'fairly' physically active compared with age peers. The perceived barriers to physical activity included work commitments, lack of leisure time and poor health. It was found that obesity, not being in work and having a limiting long-term illness associated with a lower likelihood of physical activity. Participation in sports and exercise, walking, heavy housework and gardening were all lower in non-working than working adults. Older adults had unrealistic views of their activity levels, and of work and lack of time as barriers to physical activity. It is concluded that more attention needs to be paid to health promotion and education among the over-sixties, especially those not in paid work.
\end{abstract}

KEY WORDS - physical activity, Chief Medical Officer's target, older adults, England, Health Survey for England.

\section{Background}

A physically-active lifestyle throughout the lifecourse is more beneficial to both mental and physical wellbeing than being sedentary (Nicholl, Coleman and Brazier I994). Globally, I.9 million deaths annually are attributed to physical inactivity (Ezzati et al. 2004). Participation in physical

* Department of Epidemiology and Public Health, University College London, UK. 


\section{I344 Moushumi Chaudhury and Nicola Shelton}

activity is influenced by a multitude of factors, including social, economic and cultural facets of society. Regular participation in physical activity increases quality of life and independence in older age, increases muscle strength and reduces hip fracture from falls (United States Department of Health and Human Services i996). There is abundant evidence that physical inactivity and a sedentary lifestyle are associated with an increased incidence of chronic conditions, including diabetes, obesity, cancers, osteoporosis, ischaemic heart disease (Hohl III 200I) and depression (World Health Organization 2004). These disorders become more prevalent and severe with age (Donaldson 2004). Yates et al. (2008) found that men aged 70 years in the United States of America (USA) who took up regular exercise two to four times a week, ate well, did not smoke, and maintained healthy blood pressure and normal weight, had increased longevity. The amount of habitual physical activity accrued is also closely linked with all-cause mortality risk (Blair, Cheng and Holder 200I). A recent study found those who increased activity levels between $5^{\circ}$ and 60 years of age lived as long as those who were already exercising regularly in middle age (Byberg et al. 2009), which suggests that encouraging greater participation in physical activity among older people confers significant health benefits. Cassel suggested that activity, including physical activity participation, may be 'the best treatment for aging' (2002: 2334).

Examples of such health promotion schemes that include older people in the United Kingdom are the recent free swimming scheme for the oversixties (Department of Culture, Media and Sport 2009) and the 'Lets Get Moving: Physical Activity Care Pathway' programme (Department of Health 2009). The latter identifies inactive people aged I6-74 years and offers a brief, focused intervention, which begins with administration of the General Practice Physical Activity Questionnaire (GPPAQ), and the simple four-level Physical Activity Index (PAI) that categorises participants as 'active', 'moderately active', 'moderately inactive' and 'inactive'. The GPPAQ and PAI tools are increasingly used to inform health practitioners when a brief intervention to increase physical activity is appropriate (Department of Health 2006).

Since 1997 in England, physical activity has increased among men and women in all age groups under 75 years (Chaudhury and Roth 2007), but participation declines with age. In 2006, 35 per cent of men and 27 per cent of women aged 55-64 years, compared with 2i per cent of men and I 6 per cent of women aged 65-74 years, met the current recommendation of the Chief Medical Officer for England of moderate intensity activity for $3^{0}$ minutes on at least five days a week (Chaudhury and Roth 2007). Several studies have shown that life course transitions, such as changes in employment status, have implications for physical activity participation 
(Arthur and Finch 1999; Coakley and White 1992; Robertson 2003). Lifechanging experiences, such as retirement, may encourage older adults to reconsider many aspects of their life, including their health. An analysis of cross-sectional data found large reductions in physical activity among men around the time of retirement, and that those who were retired and aged 65-74 years were 23 per cent less likely to meet the physical activity target than those aged 55-64 years (Allender et al. 2006). Berger et al. (2005) reported that employment involved a considerable amount of physical activity but it was not replaced by those who retired. Those not in work reported more physical activity at home or in leisure time than the employed, but insufficient to compensate for the loss of work-related activity, and a higher proportion participated in little physical activity outside the home.

This paper examines the reasons for the decline in physical activity among those aged 6o-69 years. We ask what proportion of men and women in the age group take regular exercise and what are the risk factors for inactivity. The paper was inspired by the appearance as a contestant of the political commentator, John Sergeant, on the 2008 BBC television programme Strictly Come Dancing (the equivalent of Dancing with the Stars in the USA). The spectacle of an overweight man around retirement age dancing (albeit badly) on national television captured the imagination of the general public. He claimed to have lost two stone (I2.7 kilograms) in weight through the regular and demanding physical activity. If he became a role model for men in their sixties, and encouraged them to increase their physical activity and reduce weight, then his departure from primetime television was a great loss. We ask how much physical activity adults of his age group do, what types of activity they pursue, why they do not do more, and what are the perceived and actual barriers to physical activity among older adults.

\section{Methods}

\section{The sample}

The reported analysis draws on data from the cross-sectional, populationbased Health Survey for England (HSfE), an annual nationwide survey of the English population living in private households. Each wave uses a Postcode Address File to recruit a stratified, two-stage, random sample that is representative of the socio-demographic profile of the English population, as more fully explained elsewhere (Craig and Mindell $2008 a$; Craig and Shelton 2008). For this paper, an analysis sample was drawn from the HSfE surveys in 2006 and 2007, comprising I,550 respondents 
aged 6o-69 years in 2006 and 56r adults aged 6o-64 years in 2007 (the pertinent questions were not asked of those aged 65 or more in 2007). In both years, questionnaires were administered in the participants' homes by trained interviewers. In addition, the HSfE 2006 asked the respondents to complete self-completion questionnaires. Over 99 per cent of those aged 6o-69 years in 2006 answered the questions on physical activity, and Ioo per cent of those aged 6o-64 years in 2007 completed the selfcompletion questionnaire on knowledge of, attitudes about and the practice of physical activity. The measures are described in more detail below. ${ }^{1}$

\section{Measurements}

The HSfE 2006 collected data on participation in physical activity during the previous four weeks, including in heavy housework, manual work, gardening, 'do-it-yourself' (DIY) home improvements, walking, and sports and exercise. The respondents were shown a card list of common sports and physical activities, such as swimming, cycling, gym workout, aerobics, keep-fit, dancing, running/jogging, football/rugby, badminton/ tennis, squash and calisthenics. The information was recorded using a computer-aided personal interview (CAPI) package. Data were also collected on smoking status, alcohol consumption and self-reported health. Height was measured with a stadiometer and weight by a scale, from which the Body Mass Index (BMI) was calculated. ${ }^{2}$ Waist and hip circumferences, as indicators of overweight and obesity, were measured by a nurse. A wide range of own and household socio-economic and socio-demographic data were also collected during the interview. A more detailed description of the methodology is available, and the HSfE recognises that self-report measures are potentially prone to response and recall bias (Craig and Mindell 2008b).

The respondents were classified into 'high', 'low' and 'medium' levels of reported physical activity. A high level was defined as 20 or more occasions of moderate or vigorous activity of at least 30 minutes duration during the previous four weeks (on an average of at least five days each week). This level of activity is the minimum required to gain some general health benefits (e.g. reduced relative risk for cardiovascular morbidity) and corresponds to the Chief Medical Officer's target (Donaldson 2004). A medium level was defined as $4^{-19}$ occasions of moderate or vigorous activity of at least 30 minutes during the last four weeks (on average on between one and four days each week). A low level was defined as up to three occasions of moderate or vigorous activity of at least 30 minutes during the last four weeks (on average on less than one day a week). 
The self-completion questionnaire administered in HSfE 2007 was specifically to obtain information about the respondents' knowledge of and attitudes to four areas of lifestyle behaviour: smoking, drinking, eating and physical activity. The module on physical activity had questions on knowledge, attitudes, social influences, barriers to and factors that encouraged physical activity (Blair, Cheng and Holder 200I; Health Education Authority ig96; Office for Official Publications of the European Communities I999). There were also questions about the respondent's knowledge of the current recommended physical activity target, their perception of how active they were compared to others of their age, their desire to be more physically active, the barriers that impeded more physical activity, and the factors that would encourage them to engage more in exercise or sport. The new questionnaire underwent cognitive testing prior to being piloted in autumn 2006. It was administered by interviewers to people aged $16-64$ years.

\section{Statistical analysis}

A logistic regression was carried out of variations in the level of physical activity among the sample (n.b. all aged 6o-69 years), with the outcome or dependent variable being whether or not the respondent's level of physical activity attained the Chief Medical Officer's target. The following covariates were included in the initial model: equivalised household income quintiles, socio-economic status using the five category National Statistics Socio-economic Classification (NS-SEG) (managerial and professional, small employers and own account workers, lower supervisory and technical and semi-routine and routine occupations) (Office of National Statistics 2002), economic-activity status (in paid employment or not), limiting long-term illness (none, non-limiting long-term illness, and limiting long-term illness), BMI (<25 kg/m², $25^{-29.9} \mathrm{~kg} / \mathrm{m}^{2}$ (overweight), $\geqslant 30 \mathrm{~kg} / \mathrm{m}^{2}$ (obese)), age in single years, waist circumference (raised waist circumference $>\mathrm{IO} 2$ centimetres $(\mathrm{cm})$ in men and $>88 \mathrm{~cm}$ in women), waist-hip ratio, smoking status (current smoker, ex-smoker, never smoked), alcohol consumption on heaviest drinking day (below maximum recommended units, above maximum units, more than twice recommended maximum units), National Health Service region, and the 2004 index of multiple deprivation (IMD) quintile for the respondent's residential area (which measures seven domains related to: income deprivation; employment deprivation; health deprivation and disability; education, skills and training deprivation; barriers to housing and services; living environment deprivation; and crime) (Communities and Local Government 2004). ${ }^{3}$ 
T A B L E I. Awareness and knowledge of current recommendation for physical activity and perception of own physical activity, adults aged 6o-64 years, England, 2007

\begin{tabular}{lcc}
\hline Variable & Men (\%) & Women (\%) \\
\hline Awareness of physical activity recommendations: & & \\
Thought they knew the recommended level of physical activity & 23 & 32 \\
Heard of recommended level of physical activity but not sure what it is & 40 & 36 \\
Had not heard about recommended level of physical activity & 37 & 32 \\
Knowledge of physical activity recommendations: & & \\
Equivalent to current minimum recommendations & 3 & 7 \\
More than current minimum recommendations & 24 & 25 \\
Less than current minimum recommendations or did not know & 73 & 68 \\
'I can get enough physical activity in daily life': & & \\
Strongly agree/agree & 57 & 57 \\
Neither agree nor disagree/can't choose & 20 & I8 \\
Strongly disagree/disagree & 24 & 24 \\
Self-perception of own physical activity: & & \\
Very and fairly physically active & 78 & 77 \\
Not very physically active & I7 & I4 \\
Not at all physically active & 5 & 9 \\
Sample size & 244 & 317 \\
\hline
\end{tabular}

Note: I. The recommendation is $30+$ minutes on five or more days a week.

\section{The descriptive results}

Among the respondents aged 6o-64 years, 23 per cent of men and 32 per cent of women thought they knew the national physical activity targets for adults, but actually few did so (3\% of men and $7 \%$ of women). Around one-quarter over-estimated the targets and the rest either did not know them or under-estimated them (see Table I). Over one-half $(57 \%)$ of the sample thought they could get enough physical activity in their daily life, and more than three-quarters (78\% of men and $77 \%$ of women) thought they were physically active compared to others of their own age. The most important perceived barriers to being more physically active were work commitments (22\% of men and I3\% of women), lack of leisure time (21 \% of men and $13 \%$ of women), and poor health $(25 \%$ of men and $22 \%$ of women). In addition, 22 per cent of the women said that they were not 'the sporty type', 4 per cent of men and 8 per cent of women said that they were too overweight, and I 2 per cent of men and 8 per cent of women said that they were too old to do more (Figure I).

\section{The regression results}

Table 2 shows the results of the logistic regression analysis of the factors that influenced physical activity participation among those aged 


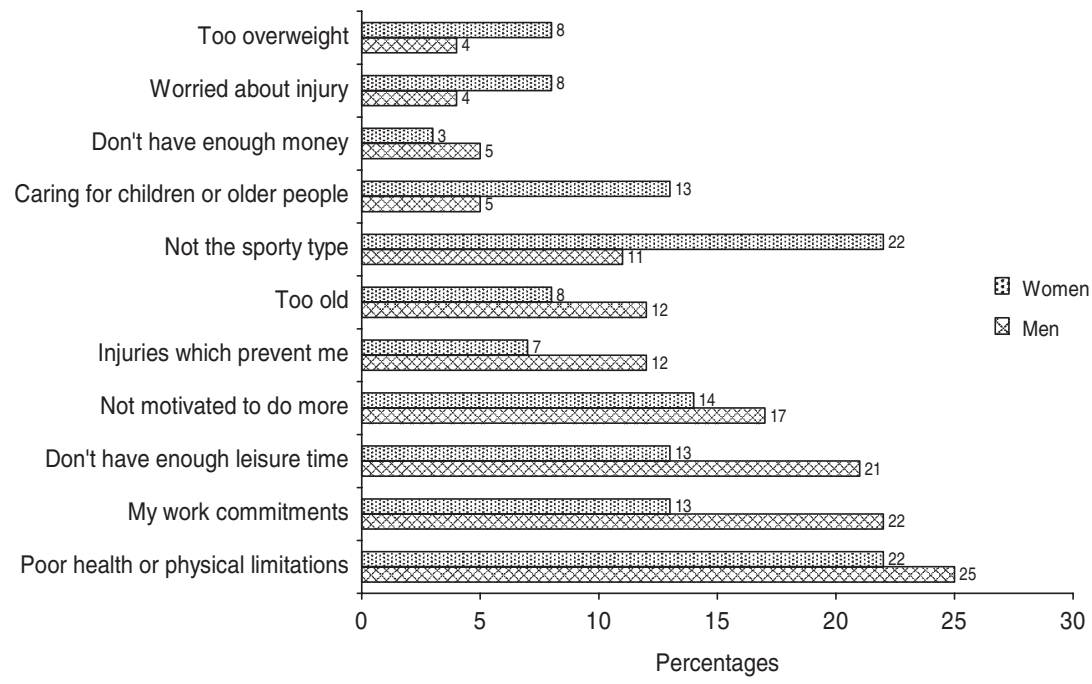

Figure I. Barriers of adults aged 6o-64 to doing more physical activity, exercise or sport by age and sex.

6o-69 years. Only variables that were statistically significant at the 5 per cent level were included in the final models. An odds ratio (OR) greater than I.o shows an increased likelihood of physical activity participation. Being in paid work was found to be associated with physical activity for both men and women. Those who were not working had odds that were about one-third of those for working adults $(\mathrm{OR}=0.37$ men, $\mathrm{OR}=0.36$ women) of participation in the target level of physical activity (Model I). After controlling for poor health and BMI, those who were not working had less than one-half the odds of working adults $(\mathrm{OR}=0.46 \mathrm{men}$, $\mathrm{OR}=0.4^{2}$ women) (Model 2). Limiting long-term illness (illness that limits activities) was also associated with lower odds of meeting physical activity targets, with again similar odds for men $(\mathrm{OR}=0.3 \mathrm{I})$ and women $(\mathrm{OR}=0.25)$. Both overweight and obesity also associated with lower levels of physical activity in men and women. Obese men $(\mathrm{OR}=0.5 \mathrm{I})$, overweight women $(\mathrm{OR}=0.63)$ and obese women $(\mathrm{OR}=0.26)$ were less likely to participate than those who were not overweight. Equivalised household income (NS-SEC), occupational status, age (in single years), waist circumference, waist-hip ratio, smoking status, alcohol consumption, NHS region and the index of multiple deprivation quintile were excluded from the final models as not statistically significant for men or women.

Given the importance of working status on physical activity, we examined its influence on participation in sports and exercise, walking, heavy manual work and heavy housework and gardening. There were no 
T А в L E 2. Estimated odds ratios for physical activity that meets Chief Medical Officer's recommendation by associated risk factors and gender, 6o-69-years-olds, England, 2006

\begin{tabular}{|c|c|c|c|c|}
\hline \multirow[b]{2}{*}{ Variable } & \multicolumn{2}{|c|}{ Men } & \multicolumn{2}{|c|}{ Women } \\
\hline & Odds ratio & $95 \%$ CI & Odds ratio & $95 \%$ CI \\
\hline \multicolumn{5}{|l|}{ Model I: } \\
\hline \multicolumn{5}{|l|}{ Employment status: } \\
\hline Employed & I.0O & & I.00 & \\
\hline Not employed & 0.37 & $0.26-0.53^{* * *}$ & 0.36 & $0.25^{-0.51^{* * *}}$ \\
\hline \multicolumn{5}{|l|}{ Model 2: } \\
\hline \multicolumn{5}{|l|}{ Employment status: } \\
\hline Employed & I.00 & & I.00 & \\
\hline Not employed & 0.46 & $0.3^{2-0.67 * * *}$ & $0.4^{2}$ & $0.28-0.6 \mathrm{I} * * *$ \\
\hline \multicolumn{5}{|l|}{ Long-term illness: } \\
\hline None & I.00 & & I.OO & \\
\hline Non-limiting & І.06 & $0.7 \mathrm{I}-\mathrm{I} .5^{8}$ & I.08 & $0.70-1.68$ \\
\hline Limiting & $0.3 \mathrm{I}$ & $0.19^{-0.50^{* * *}}$ & 0.25 & $0.15^{-0.43^{* * *}}$ \\
\hline \multicolumn{5}{|l|}{ Body Mass Index: } \\
\hline$<25 \mathrm{~kg} / \mathrm{m}^{2}$ & I.OO & & I.OO & \\
\hline$\geqslant 25-29.9 \mathrm{~kg} / \mathrm{m}^{2}$ (overweight) & $0.7 \mathrm{I}$ & 0.49-I.09 & 0.63 & $0.4 \mathrm{I}-0.96^{*}$ \\
\hline$\geqslant 30 \mathrm{~kg} / \mathrm{m}^{2}$ (obese) & $0.5 \mathrm{I}$ & $0.3 \mathrm{I}^{-0}-85^{* * *}$ & 0.26 & $0.15^{-0.45^{* * *}}$ \\
\hline Not measured & 0.57 & $0.30^{-1.10}$ & $0.4^{2}$ & $0.22-0.78^{* *}$ \\
\hline Sample size & 715 & & 834 & \\
\hline
\end{tabular}

Notes: The Chief Medical Officer's recommendation is $30+$ minutes on five or more days a week. CI: confidence interval.

Significance levels: ${ }^{*} p<0.05,{ }^{* *} p<0.01,{ }^{* * *} p<0.001$.

significant differences in sports and exercise participation for most activities for men and women in this age group (see Table 3). Swimming, doing exercises (e.g. press-ups and sit-ups) and yoga were significantly lower among non-working women, and swimming, running and jogging were significantly lower among non-working men. The level of participation in none of the specified sports was not significantly higher among the respondents not in work. Walking (I5 or more minutes at a brisk fast pace) was also significantly lower in men and women who were not working. Men were much more likely than women to be engaged in non-occupational heavy manual work (e.g. DIY), but there were no differences by work status. Participation in moderate levels of heavy housework and gardening were significantly lower in women who were not working, but not among men.

\section{Discussion}

Those aged 6o-69 years who responded to the Health Survey for England in 2006-07 thought that work and lack of leisure time were barriers to 
T A B L E 3. Participation in activities during the last four weeks by gender and work status, 6o-69-year-olds, England, 2006

\begin{tabular}{|c|c|c|c|c|}
\hline \multirow[b]{2}{*}{ Activity } & \multicolumn{2}{|r|}{ Men } & \multicolumn{2}{|c|}{ Women } \\
\hline & In work & Not working & In work & Not working \\
\hline & \multicolumn{4}{|c|}{ Percentages } \\
\hline \multicolumn{5}{|l|}{ Individual sports: } \\
\hline Swimming & II & 9 & I7 & I2 \\
\hline Cycling & 9 & 9 & 2 & 3 \\
\hline Workout gym/exercise bike/weight training & 8 & 7 & 7 & 6 \\
\hline Exercise (e.g. press ups, sit ups) & 7 & 7 & II & 8 \\
\hline Running, jogging & 7 & 2 & 7 & 6 \\
\hline Any other type of dancing & 4 & 3 & 2 & o \\
\hline Aerobics, keep fit, gymnastics, dance for fitness & 3 & I & 7 & 6 \\
\hline Football, rugby & I & I & 0 & 0 \\
\hline Golf & 7 & 7 & 2 & I \\
\hline Bowls (indoor, outdoor, crown, green), petanque & 2 & 3 & 0 & 2 \\
\hline Fishing, fly fishing & I & I & $\mathrm{o}$ & o \\
\hline Yoga & o & o & 3 & I \\
\hline Any sports participation & 34 & 24 & 32 & 25 \\
\hline \multicolumn{5}{|l|}{ Other physical activity: } \\
\hline Walking ( $\mathrm{I}_{5}+$ minutes at brisk/fast pace) & $3^{8}$ & 27 & 33 & 19 \\
\hline Any heavy manual & 40 & 34 & I6 & I3 \\
\hline $\begin{array}{l}\text { Heavy housework, gardening, do-it-yourself } \\
\text { repairs, improvements (moderate levels) }\end{array}$ & 63 & 62 & 73 & 62 \\
\hline Sample size & 715 & & 834 & \\
\hline
\end{tabular}

physical activity, but the inverse was found to be the case. There were no sports with significantly higher participation among those who were not working (who presumably had more leisure time). Physical limitations were a barrier to participation, but employment was not. Working was equally important for both men and women, even given the lower level of employment among women. Being in employment may provide income to pay for participation in various types of physical activity, such as gym, pool and club memberships or individual sports sessions.

It is likely that opportunities for occupational and non-occupational physical activity are reduced when no longer employed. There is likely to be less occupational physical activity, especially for those who were manually employed, and other influences may be less physical activity associated with walking or cycling to and from work, smaller social networks and associated activities, less participation with colleagues in team sports or, say, dancing, and less access to facilities, e.g. workplace gyms, swimming pools and sports grounds. The lower levels of walking in the non-working group were particularly marked. This suggests that schemes such as free swimming for the over-sixties, whilst removing a financial barrier, only partially compensate for work-related losses and do not make 
access to facilities easier. Schemes that provide transport (possibly including free bicycles given the relative popularity of cycling), team-sports' facilities and group activities with social and physical dimensions such as walking groups or conservation projects might have beneficial results.

From a public health perspective, there is a need to recognise both the lack of knowledge in the population of what constitutes sufficient physical activity and the reduction in participation in physical activity that tends to occur when people retire from work. General practitioners should provide information on and encourage continued physical activity participation in the local area and beyond, explain the Chief Medical Officer's targets, and stress the multiple health benefits. Given that after controlling for health status, obese men and women were less likely to participate in physical activity than others, they need to be targeted for health promotion education and interventions, and there is also a case for health promotion strategies that specifically focus on the population of retirement age.

This study has presented evidence for those aged 6o-69 years in the general population of England, but only a small proportion of the sample were members of the country's growing minority ethnic groups. Stamatakis (2005) reported from the HSfE 2004 that for men and women aged 55 or more years, physical activity participation was lower in most non-white ethnic groups compared to white groups - particularly the Indian, Pakistani, Bangladeshi and Chinese-origin populations but not Black-Caribbeans. This suggests that specific interventions tailored for these groups may be needed to boost their participation in physical activity. Studies have shown that the terms 'activity' and 'exercise' mean different things for people of different cultural backgrounds. We concur with Bird et al. (2009) that more research is needed to design and implement physical activity promotion interventions that are culturallytailored and sensitive.

To the best of our knowledge, the reported analysis is the first in England to have examined the attitudes towards, perceptions of and knowledge about physical activity and its benefits among those aged 6o-64 years using a nationally-representative sample. Large cross-sectional surveys such as the HSfE provide robust evidence of the levels of participation in physical activity and of variations by age, gender and health status, but do not allow us to specify the causal relationships, such as how adults adopt, maintain or cease to participate in sport and physical activity throughout their lives (Allender, Cowburn and Foster 2006; for a Spanish study with a longitudinal dimension see Castillo et al. 20Io). Those who were obese may be so through not participating in physical activity, rather than their not participating in physical activity through being obese. Obesity might be a barrier through negative perceptions of body image, especially for women. 
Indeed, a few respondents reported that they were too overweight to do more, and some women also reported that they were not the 'sporty type'. Similar circular reasoning may be used by people with limiting long-term illnesses to account for their low physical activity participation.

It is evident that many more people think they know the current recommendations for adults about the level of beneficial physical activity than actually do, and that many people have unrealistic views of their own levels of activity. Being in paid work associates with higher levels of physical activity regardless of health status. More health education around physical activity targets and more health promotion interventions are needed for the over-sixties who are no longer in work. Being of retirement age does not seem to change the perception of physical activity in adults but it does impact on how physically active individuals are.

\section{Acknowledgements}

We are grateful to the participants in the Heath Survey for England (HSfE), its field staff and our colleagues at the National Centre for Social Research (NatCen). The HSfE 2006 and 2007 were funded by the National Health Service Information Centre for Health and Social Care. The funder played a substantial role in determining the content of the surveys but played no part in the design, analysis or interpretation of this study, in drafting this article or the decision to submit for publication. The authors' work is independent of the funders, and we were responsible for conception, design, analysis and interpretation. We thank Dr Aparna Shankar for her comments on earlier drafts. The views expressed in this article are those of the authors and do not necessarily reflect those of the funder.

\section{NOTES}

I Ethics approval for the Health Survey for England was gained from the London Multi-Centre Research Ethics Committee (MREG).

2 Body Mass Index is given by the formula (height in metres/weight in kilograms squared). It is a widely recognised indicator of overweight and obesity (National Institute of Health and Clinical Excellence 2006; World Health Organization 2009).

3 Since 2003, HSfE has closely analysed non-response bias and generated weights that correct when producing population estimates: the details are available in Craig and Mindell $(2008 a, 2008 b)$. These were routinely used in the reported analyses. The descriptive analysis used SPSS version I5, and the logistic regression analysis used STATA ro, using weighting and taking into account sampling framework. The HSfE 2007 adopts a clustered, stratified multi-stage sample design. One of the effects of using the complex design and weighting is that standard errors for survey estimates are generally higher than the standard errors that would be derived from an unweighted simple random sample of the same size. 


\section{References}

Allender, S., Cowburn, G. and Foster, C. 2006. Understanding participation in sport and physical activity among children and adults: a review of qualitative studies. Health Education Research, 21, 6, 826-35.

Allender, S., Peto, V., Scarborough, P. and Rayer, M. 2006. Diet, Physical Activity and Nutrition. British Heart Foundation, London.

Arthur, S. and Finch, H. 1999. Physical Activity in Our Lives: Qualitative Research among Disabled People. Health Education Authority, London.

Berger, U., Der, G., Mutrie, N. and Hannah, M. H. 2005. The impact of retirement on physical activity. Ageing \& Society, 25, 2, I8I-95.

Bird, S., Radermacher, H., Feldman, S., Sims, J., Kurowski, W., Browing, C. and Thomas, S. 2009. Factors influencing the physical activity levels of older people from culturally-diverse communities: an Australian experience. Ageing \& Society, 29, 8, I275-94.

Blair, S. N., Cheng, Y. and Holder, S. 200I. Is physical activity or physical fitness more important in defining health benefits? Medicine and Science in Sports and Exercise, 33, 6, $\mathrm{S}_{379}-99$.

Byberg, L., Melhus, H., Gedeborg, R., Sundström, J., Ahlbom, A., Zethelius, B., Berglund, L. G., Wolk, A. and Michaëlsson, K. 2009. Total mortality after changes in leisure time physical activity in 50 year old men: 35 year follow-up of population based cohort. British Medical fournal, 338, 5 March, b688.

Cassel, C. K. 2002. Use it or lose it: activity may be the best treatment for aging. Fournal of the American Medical Association, 288, I8, 2333-5.

Castillo, J. M. del, Navarro, J. E. J.-B., Sanz, J. L. G., Rodríguez, M. M., Izquierdo, A. C. and Pinés, D. del H. 2010. Being physically active in old age: relationships with being active earlier in life, social status and agents of socialization. Ageing E Society, 3o, 7 , I099-II5.

Chaudhury, M. and Roth, M. 2007. Cardiovascular diseases and risk factors in adults. In Craig, R. and Mindell, J. M. (eds), The Health Survey for England 2006. Health and Social Care Information Centre, Department of Health, Leeds, UK, II I-34.

Coakley, J. and White, A. I992. Making decisions: gender and sport participation among British adults. Sociology of Sport fournal, 9, I, 20-35.

Communities and Local Government 2004. The English Indices of Deprivation 2004: Summary. Revised edition, Communities and Local Government, London. Available online at http://www.communities.gov.uk/documents/communities/pdf/I31206.pdf [Accessed 27.June 2009].

Craig, R. and Mindell, J. (eds) 2008 . The Health Survey for England 2006: CVD and Risk Factors for Adults, Obesity and Risk Factors for Children. Health and Social Care Information Centre, Department of Health, Leeds, UK.

Craig, R. and Mindell, J. (eds) 2008 b. The Health Survey for England 2006. Volume 3, Methodology and Documentation. Health and Social Care Information Centre, Department of Health, Leeds, UK.

Craig, R. and Shelton, N. J. (eds) 2008. The Health Survey for England 2007. Volume 3, Methodology and Documentation. Health and Social Care Information Centre, Department of Health, Leeds, UK.

Department of Culture, Media and Sport 2009. Free Swimming Programme. Department of Culture, Media and Sport, London.

Department of Health 2006. The General Practice Physical Activity Questionnaire (GPPAQ): A Screening Tool to Assess Adult Physical Activity Levels Within Primary Care. Department of Health, London. 
Department of Health 2009. Let's Get Moving: Physical Activity Care Pathway. Department of Health, London.

Donaldson, L. 2004. At Least Five a Week: Evidence on the Impact of Physical Activity and its Relationship to Health. Chief Medical Officer, Department of Health, London.

Ezzati, M., Lopez, A., Rodgers, A. and Murray, C. (eds) 2004. Comparative Quantification of Health Risks: Global and Regional Burden of Disease Due to Selected Major Risk Factors. World Health Organization, Geneva.

Health Education Authority 1996. Promoting Physical Activity in Primary Health Care: Guidance for the Primary Healthcare Team. Health Education Authority, London.

Hohl III, H. W. 200I. Physical activity and cardiovascular disease: evidence of a dose response. Medicine and Science in Sports and Exercise, 33, 6, $\mathrm{S}_{472-83}$.

National Institute of Health and Clinical Excellence (NICE) 2006. Obesity: The Prevention, Identification, Assessment and Management of Overweight and Obesity in Adults and Children. NICE, London.

Nicholl, J. P., Coleman, P. and Brazier, J. E. 1994. An assessment of the health and healthcare costs and benefits of exercise. Pharmacoeconomics, 5, 2, I09-22.

Office for Official Publications of the European Communities i999. A Pan-EU Survey on Consumer Attitudes to Physical Activity, Body Weight and Health. Office for Official Publications of the European Communities, Luxembourg.

Office of National Statistics 2002. The National Statistics Socio-economic Classification. Office of National Statistics, London. Available online at http://www.ons.gov.uk/aboutstatistics/classifications/current/ns-sec/index.html [Accessed 27 June 2009].

Robertson, S. 2003. 'If I let a goal in, I'll get beat up': contradictions in masculinity, sport and health. Health Education Research, 18, 6, 706-16.

Stamatakis, E. 2005. Physical activity. In Sproston, K. and Mindell, J. M. (eds), The Health Survey for England 2004. Health and Social Care Information Centre, Department of Health, Leeds, UK, 237-6r.

United States Department of Health and Human Services 1996. Physical Activity and Health: A Report of the Surgeon General. Center for Disease Control, Atlanta, Georgia.

World Health Organization 2004. Global Strategy on Diet, Physical Activity and Health. World Health Organization, Geneva.

World Health Organization 2009. Global Database on Body Mass Index (BMI) Classification. World Health Organization, Geneva. Available online at http://apps.who.int/bmi/ index.jsp?introPage $=$ intro_3.html [Accessed i7 June 2009].

Yates, L. B., Djoussé, L., Kurth, T., Burning, J. E. and Gaziano, J. M. 2008. Exceptional longevity in men: modifiable factors associated with survival and function to age 9o years. Archives of Internal Medicine, 168, 3, 284-9o.

Accepted I7 May 20Io; first published online 26 August 2010

Address for correspondence:

Moushumi Chaudhury, Department of Epidemiology and Public Health, University College London, I-9 Torrington Place, London WGiE 6BT, UK.

E-mail: moushumi.chaudhury@ucl.ac.uk 